\title{
Research on Two - Way Interactive Communication and Information System Design Analysis
}

\author{
Dong $\mathrm{Xu}^{1, \mathrm{a}}$ \\ ${ }^{1}$ Chongqing College of Electronic Engineering, Chongqing, China, 401331 \\ ${ }^{a}$ email,
}

Keywords: Power Grid; Users; Two-Way Interaction; Communication and Information; System Design

\begin{abstract}
With the rapid development of social economy, China's urbanization and industrial construction process is accelerating, people's living standards and quality has also been significantly improved in the daily life of the use of electrical equipment also increased, which is to a certain extent on the increase in the power grid construction requirements. Coupled with the increase in demand for information, in the power system is also gradually achieve two-way interaction to promote better communication between the grid and users, which meet the needs of users at the same time can also promote the further development of power grid construction. This paper mainly analyzes and studies the three aspects of two-way interactive communication and information technology of power grid and users, two-way interactive communication and information system analysis of power grid and users, and two-way interactive communication and information system design of power grid and users. The better design of the grid and the user two-way interactive communication and information system provides a valuable reference.
\end{abstract}

\section{Introduction}

At present, China is committed to improving the quality of power supply services, the improvement of power grid operation efficiency, energy saving and emission reduction, the development of new energy, and gradually attach importance to the development of intelligent construction of power grid, comprehensive improvement of power grid interaction, automation, digital and information degree. Which is the interaction between the grid and the user through the corresponding electronic terminal to achieve two-way interaction and communication, according to advanced science and technology between the two can achieve real-time connectivity and network interaction, which helps to improve the user in the power construction of the degree of participation, but also to achieve battery integrated charging, remote home appliance control and other important means of business.

\section{The Grid and the User Two-Way Interactive Communication and Information Technology}

The Intelligent Electricity Technology Needs. In the traditional power grid, because it is mainly one-way communication, low degree of intelligence, resulting in the lack of communication between the grid and the user, even if there is communication, then only the user to the user one-way transmission control information, so that the grid business It is difficult to pay the arrears, peak and valley electricity prices, power supply and demand balance and other related information sent to the user, the user can not use the electrical equipment when the mode of operation to adjust, smart electricity real-time, security and reliability requirements It is difficult to be satisfied. In the technical aspects of the problems are mainly reflected in three aspects: First, the demand for communication networks. In the construction of intelligent power system, it also said that its commitment to more business, such as triple play, management information system, interactive marketing system, which also increased the requirements of the communications broadband, but in the user a Side, communication network resources are still relatively scarce, but also need to strengthen the communication network expansion measurement establishment and use [1]. For the user service, especially the large users, the original application of centralized meter reading system 
and load management, there is a low level of intelligence, low degree of standardization issues, the user's massive information needs are difficult to meet; at the same time in the personalized service promotion In the study is not enough depth, resulting in interactive means and service content is also relatively scarce; users and power systems are not fully interactive between the function cannot share the professional information system resources data, the urgent need to build a professional power platform. Although the current electric charge and discharge service system has been implemented, but in the actual application process there are still many problems, especially to achieve energy between the two-way conversion has yet to be further strengthened, while the national government should also be in response to demand, Policy and other aspects to provide policy support, so as to promote its rapid development.

The Two-Way Interactive Intelligent Electricity Technology. In the smart electricity technology, including smart meters, two-way interaction, intelligent collection, high-speed real-time communications, etc., the full combination of advanced measurement technology, modern communications technology and computer application technology.

Through the use of intelligent interactive terminal, power grid and the user can be a very good interaction between, and the use of this platform can also be achieved visual. Intelligent interactive terminal as a visualization equipment, mainly the user internal installation, through the smart meter to complete the information transceiver, but also to achieve home appliances communications, power outages, electricity and other information to remind the information displayed and provide the corresponding value-added services [3]. Intelligent interactive terminal as an important intelligent power support technology, not only to achieve effective interaction between each other, but also more accurate and timely scheduling.

In the two-way interactive system, the most important is the need to strengthen the construction of communication systems, two-way interactive system for the flow of information and business flow to provide carrier, to ensure the normal operation of the system on the basis of the grid and the user between the two-way interactive. Through the construction of communication backbone network, power optical fiber technology can be very good as a communication channel, and in the construction of access network, strengthen the wireless public network, wireless network, power line communication and fiber optic network application [3]. For the user, you can use the existing telephone lines, power lines and other equipment to build the internal network, select the communication.

\section{The Grid and the User Two-Way Interactive Communication and Information System Analysis}

The System Framework. For the interactive intelligent electricity service system, its main role is to achieve the power grid and the user before the interaction, through the full consideration of existing communication technology, intelligent home, smart meter and other facilities as the basis for the two-way interactive implementation to provide technical support. At the same time in order to meet the needs of the user's intelligent electricity service, power grid and users between the business flow, energy flow, information flow between the interaction, it should strengthen the application of the relevant platform. And the construction of intelligent interactive terminal, to improve the level of visualization in the interaction, so that we can meet the community services, triple play, smart home and other value-added services. Among the interactive intelligent electricity service system, it mainly include the communication network platform, interactive intelligent electricity service system interactive terminal, interactive intelligent electricity service system application platform [4].

The communication network platform is mainly for the exchange of information and transmission to provide protection to ensure that the two-way interactive system communication network of two-way efficient, stable and reliable and flexible. Generally in the construction process will give priority to select a dedicated network, if the conditions are not allowed, then you can also use the public network instead. One of the communication network can be divided into local access network and remote communication network, the local access network refers to the main 
low-voltage fiber composite cable, RS485 bus, wireless sensor network, micro-power wireless, remote communication network refers to the main GPRS, Wireless private network, optical fiber and other Internet and wireless public network.

The terminal as a grid and the user to achieve two-way interaction between the user to provide diversified, intelligent service of the important infrastructure platform, which mainly includes smart appliances, smart meters, intelligent interactive terminals and other parts, to be collected User information uploaded to the power platform which, and the use of 95598 power supply service center, online business hall, intelligent interactive terminal, etc. to achieve the user payment, business acceptance, information services and other services [5].

Its role is mainly for the marketing business and two-way interactive services to provide applications for intelligent services to provide an important support. In the background of the main station system, including auxiliary and marketing platform, intelligent electricity service platform and electricity information collection platform, through the system analysis and processing of electricity data information, the user feedback value-added services and electricity guidance.

The Intelligent Electricity Information Interactive Platform. Auxiliary analysis and decision-making platform, marketing business service platform, intelligent electricity service platform, electricity information collection platform is an important part of intelligent electricity information interactive platform, in which the basic application system is intelligent user service platform and electricity information collection platform, Mainly to collect and monitor the corresponding electricity service information; advanced application system is aided analysis and decision-making platform for the corresponding decision-making services. In the information interactive platform, there are more business and information exchange, the collection object mainly includes resident users, industrial and commercial users and large users, on-line monitoring device can carry out real-time collection of important information, such as user load, voltage and power, To ensure the integrity of the underlying information. In the collection of electricity information mainly includes charge and discharge and energy storage system, intelligent meter management system, distributed power management system and other applications, through the centralized processing of electricity information, so that we can further optimize the management of distributed power access to improve the degree of intelligent measurement services [6].

\section{The Grid and the User Two-Way Interactive Communication and Information System Design}

The Two-Way Interactive Service System Networking Program. By enabling two-way interaction between the grid and the user, it is possible to guide and encourage the user to participate better in power system management and operation. In interactive use of intelligent electricity is one of its important manifestations, but also with the traditional power grid between the important aspects of differences. On the one hand through the use of sensing devices, the grid will be able to real-time monitoring of the operation of power equipment, and data integration analysis, so that electricity can be used during the peak period of power supply to make a unified adjustment to ensure that all aspects of demand enough to get very good to meet; on the other hand in the grid management to encourage more users to participate in, thereby improving the quality of electricity services, reduce energy consumption to achieve the common development of environment and electricity [7]. Generally in the two-way interactive system network, including the terminal layer, network layer and application layer three levels.

The Intelligent Community Networking. Intelligent power supply district is an emerging application in recent years, is powered by the enterprise-led, able to provide users with online payment, intelligent meter reading, marketing interaction, broadband Internet access, voice, video and other services, and by means of power communication network to fully integrate the business. The current home access mode mainly uses fiber, EPON technology to provide technical support, and in the intelligent community building interchange location placed ONU, which means that the way to achieve EPON technology FTTB. For FTTB way, its scope of application is mainly on the broadband requirements are not high, the relative number of small residential users [8].

The Family Networking. The network within the family, you can make full use of the first use 
of electricity lines and other lines, with the PLC way to home appliances and network to achieve connectivity between, if there is a new security equipment or electrical equipment, you can use wireless connection. Zigbee as a wireless two-way communication technology, which is characterized by low cost, low power consumption, low complexity, close, and home network communication needs can be a good match between. In the home network gateway, mainly the use of narrowband PLC, micro-power wireless and other means of communication, security equipment, smart sockets and other subordinate equipment for unified management which can also be more simple access to more subordinate equipment [9].

The Large User Networking. In the intelligent power supply area and large user communication mode is the same, with consistency. Which access to large users, mainly through the use of EPON technology for networking, and connect the corresponding ONU, photoelectric converters and other network equipment and conversion equipment, so that you can achieve video conferencing, OA office business. In the design of large user networking, its basis and based on the main information interaction, and its interaction to the interaction and energy interaction, in reducing the cost of large users of colleagues, to provide the corresponding value-added services. In the large user internal information collection, mainly including communication, power grid and large user three parts, when the information collection is completed after the use of the network to the main station system transmission, after analysis will be converted into the corresponding power marketing information and Power guidance information is transmitted to the user. In the main station system, its main role is to develop Web software system, acquisition system and marketing system, and through the Web form to large users to publish information, the advantage is as long as the user in the relevant equipment installed after the client, are able to get the corresponding information.

\section{Conclusion}

In general, the two-way interaction between the grid and the user information and communication system is an important aspect of the construction of smart grid, but also can better meet the power grid integrated service capabilities, interactive marketing needs, information synchronization, real-time interactive response And other functions, which improve the power grid construction automation and intelligent level have a positive role in promoting.

\section{Acknowledgements}

Fund Project: Chongqing Municipal Education Commission Science and Technology Research Project. Title: Research on the Construction and Recognition Method of Complementary Feature Collection of Face Images. Project Number: KJ1729405

\section{References}

[1] QI Bing, ZHANG Rong, LI Bin, CHEN Song-song.Design of Automatic Demand Response Information Exchange Interface [J / OL]. China Journal of Electrical Engineering, 2014 (31). Http: //www.cnki.net/kcms /detail/10.13334/j.0258-8013.pcsee.2014.31.016.html

[2] Wang Yan, Mu Hao Nan, Yang Xiaowei, Fang Jian'e. Design of Power Grid Monitoring System Based on ARM and DSP [J]. Computer Measurement and Control, 2014, 10: 3138-3140.

[3] Wang Jinming, Yang Yongbiao, Gao Kai, Li Yichao. Two-way interactive service data acquisition and control of communication technology[J]. China New Communications, 2015,15: 70.

[4] ZHU En-guo, DUAN Jian. Two-way interactive function design and key technology of electricity information acquisition system[J]. Automation of Electric Power Systems, 2015,17: 62-67. 
[5] Li Ruyi, Wang Huicai, Hou Xingzhe, Liu Yongguang, Liu Yongxiang. Design and Implementation of Interactive Energy Saving System for Intelligent Buildings[J]. Journal of Electrical Engineering, 2015, S2: 63-68.

[6] Chen Da, Yang Yongbiao, Wang Jinming, Fu Junqiang. Design of Power System and User Interaction System Based on IEC International Standard[J]. Energy Engineering, 2016, 03: 13-17.

[7] Liu Xuan, Xu Yinghui, Zhao Rui, Yang Ting. Reliable channel configuration algorithm for bidirectional interaction of electricity information[J]. Journal of Electric Power Systems and Automation, 2016, 07: 101-105 + 134 .

[8] Zhu Enguo, Fan Hong, Dou Jian, Yu Yangqi. Design and Application of Interactive Model for Electricity Information Collection System Based on SEP2 and IEC61970CIM [J]. Electrical Measurement \& Instrumentation, 2016, 21: 89-94.

[9] Tan Mei, Li Zhiyong. Intelligent community two-way interactive service system design [J]. Electrical Engineering, 2013, 11: 74-76. 